\title{
Recent Radiation Test Results for Trench Power MOSFETs
}

\author{
Jean-Marie Lauenstein, Member, IEEE, Megan C. Casey, Member, IEEE, Edward P. Wilcox, \\ Member, IEEE, Anthony M. Phan, Hak S. Kim, Alyson D. Topper, Member, IEEE, \\ Raymond L. Ladbury, Member, IEEE, and Kenneth A. LaBel, Member, IEEE
}

\begin{abstract}
Single-event effect (SEE) radiation test results are presented for various trench-gate power MOSFETs. The heavyion response of the first (and only) radiation-hardened trenchgate power MOSFET is evaluated: the manufacturer SEE response curve is verified and importantly, no localized dosing effects are measured, distinguishing it from other, non-hardened trench-gate power MOSFETs. Evaluations are made of n-type commercial and both n- and p-type automotive grade trench-gate device using ions comparable to of those on the low linear energy transfer (LET) side of the iron knee of the galactic cosmic ray spectrum, to explore suitability of these parts for missions with higher risk tolerance and shorter duration, such as CubeSats. Part-to-part variability of SEE threshold suggests testing with larger sample sizes and applying more aggressive derating to avoid on-orbit failures. The n-type devices yielded expected localized dosing effects including when irradiated in an unbiased $(0-V)$ configuration, adding to the challenge of inserting these parts into space flight missions.
\end{abstract}

\section{INTRODUCTION}

VERTICAL trench-gate style MOSFETs have been a staple commercially for low- to medium-voltage applications due to their lower on-state resistance ( $\left.\mathrm{R}_{\mathrm{DS} \_ \text {on }}\right)$. A lower $\mathrm{R}_{\mathrm{DS} \_\mathrm{ON}}$ is achieved in part from the vertically-oriented gates penetrating past the body region, thereby eliminating the parasitic JFET that limits the minimum cell pitch in planargate vertical double-diffused MOSFET (VDMOS). With the advent of CubeSats, these trench-gate MOSFETs have become common on spacecraft tolerant of higher risk. Trench-gate power MOSFETs are vulnerable to both catastrophic single-event effects (SEE) and degradation due to localized ionizing dose effects from heavy ions [1-7]. In

Manuscript received July 12, 2017. This work was supported in part by the NASA Electronic Parts and Packaging Program (NEPP), the NASA Engineering Safety Center (NESC), and International Rectifier Corporation (now Infineon, A.G.).

J.-M. Lauenstein, M. C. Casey, R. L. Ladbury, and K. A. LaBel are with NASA Goddard Space Flight Center, Code 561.4, Greenbelt, MD 20771 (USA), (email: jean.m.lauenstein@nasa.gov, megan.c.casey@nasa.gov, raymond.l.ladbury@nasa.gov, and kenneth.a.label@nasa.gov.)

E. P. Wilcox, A. M. Phan, H. S. Kim, and A. D. Topper are with ASRC Federal Space and Defense, Inc. (AS\&D, Inc.), work performed for NASA/GSFC, Code 561.4, Greenbelt, MD 20771 (USA), (email: ted.wilcox@nasa.gov, anthony.m.phan@nasa.gov, hak.s.kim@nasa.gov, and alyson.d.topper@nasa.gov).
2013, International Rectifier Corp. (now Infineon, A.G.) released the first (and to date, only) radiation-hardened trench-gate power MOSFET. It is an n-type, $20 \mathrm{~V}$, logic-level device targeted for point-of-load applications [8].

Typically safe-operating areas for SEE in power MOSFETs are established using ions with atomic number $(\mathrm{Z})>35$ and high linear energy transfer (LET) $\left(>37 \mathrm{MeV} \cdot \mathrm{cm}^{2} / \mathrm{mg}\right)$ to ensure safety in the majority of space radiation environments. In contrast, the objectives of this work are in part to evaluate non-hardened vertical trench-gate power MOSFETs with lower-LET, lighter ions relevant to higher-risk tolerant, shorter duration space missions. In addition, validation of manufacturer test results of the radiation-hardened trench-gate power MOSFET is performed and demonstrates the successful elimination of the localized dosing effects in nonhardened trench-gate power MOSFETs. Table I provides a list of all devices tested.

TABLE I

SUMMARY OF POWER MOSFETS TESTED

\begin{tabular}{|c|c|c|c|c|c|}
\hline Part \# & Manufacturer & Grade & $\begin{array}{c}\text { BVDSS } \\
(\mathrm{V})\end{array}$ & $\begin{array}{c}\mathrm{I}_{\mathrm{D}} \\
(\mathrm{A})\end{array}$ & $\begin{array}{c}\mathrm{R}_{\mathrm{DS} \text { ON }} \\
(\Omega)\end{array}$ \\
\hline \hline Si7414DN & Vishay & Commercial & 60 & 8.7 & 0.025 \\
\hline SQS460EN & Vishay & Automotive & 60 & 8 & 0.036 \\
\hline SQJ431EP & Vishay & Automotive & -200 & -12 & 0.213 \\
\hline NVTFS5116PL & ON Semi & Automotive & -60 & 14 & 0.052 \\
\hline BSS84AKV & Nexperia & Automotive & -50 & 0.17 & 7.5 \\
\hline IRHLF87Y20 & Int'l Rectifier & Rad Hardened & 20 & 12 & 0.032 \\
\hline
\end{tabular}

\section{EXPERIMENTAL METHODS}

\section{A. Heavy-Ion Testing}

Heavy-ion test data were taken at the Texas A\&M University Cyclotron Facility (TAMU) in air (1.5 cm airgap) or at Lawrence Berkeley National Laboratory (LBNL) in vacuum (Table II). All ion energies used were sufficient to place the Bragg peak past the epilayer into the substrate region of the MOSFETs. Six devices-under-test (DUTs) can be mounted on the test board with daughter cards and individually accessed via dry Reed relays controlled by an Agilent 34907A data acquisition/switch unit. All terminals of the devices not under test are then floating. The equivalent circuit of the selected device is shown in Fig. 1. Additional tests of the Si7414DN were conducted under $0 \mathrm{~V}$ drain- 
source $\left(\mathrm{V}_{\mathrm{DS}}\right)$ and $0 \mathrm{~V}$ gate-source $\left(\mathrm{V}_{\mathrm{GS}}\right)$. This bias condition was achieved either by setting the gate and drain currentvoltage sourcing and measurement units (SMUs) to $0 \mathrm{~V}$ to be able to measure current, or by tying the DUT pins together at the daughter card.

Samples were delidded or decapsulated and fully electrically characterized off-site; on-site prior to the first irradiation run and following each irradiation run, a gate stress test was performed in which the gate leakage current (IGSS) was measured as a function of gate voltage at $0 \mathrm{~V}_{\mathrm{DS}}$, and the drain-source breakdown voltage ( $\left(\mathrm{BV}_{\mathrm{DSS}}\right)$ or the drainsource leakage current at $0 \mathrm{~V}_{\mathrm{GS}}$ (IDss) was measured. Measurement equipment included a Keithley 2635A or 2400 SMU for gate voltage supply and current measurement $(<1$ nA accuracy) and a Keithley 2657A or 2410 SMU for the drain voltage supply and drain current measurement.

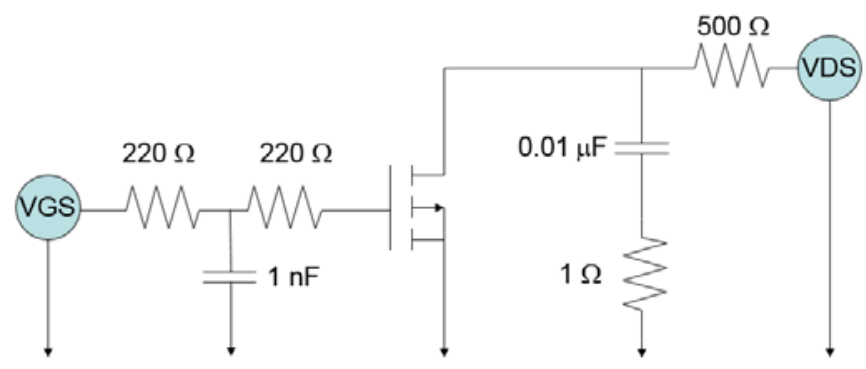

Fig. 1. Equivalent power MOSFET heavy-ion test circuit.

TABLE II

ION BEAM PROPERTIES

\begin{tabular}{|c|c|c|c|c|c|}
\hline Facility & Ion & $\begin{array}{c}\text { Air } \\
\text { Gap } \\
(\mathrm{cm})\end{array}$ & $\begin{array}{c}\text { Surface } \\
\text { Energy } \\
(\mathrm{MeV})\end{array}$ & $\begin{array}{c}\text { Surface LET } \\
\left(\mathrm{MeV} \cdot \mathrm{cm}^{2} / \mathrm{mg}\right)\end{array}$ & $\begin{array}{c}\text { Range } \\
(\mu \mathrm{m})\end{array}$ \\
\hline \hline TAMU & ${ }^{20} \mathrm{Ne}$ & 1.5 & 283 & 2.7 & 279 \\
\hline TAMU & ${ }^{40} \mathrm{Ar}$ & 1.5 & 548 & 8.2 & 202 \\
\hline LBNL & ${ }^{40} \mathrm{Ar}$ & 0 & 400 & 9.7 & 130 \\
\hline LBNL & ${ }^{65} \mathrm{Cu}$ & 0 & 659 & 21 & 108 \\
\hline LBNL & ${ }^{86} \mathrm{Kr}$ & 0 & 886 & 31 & 110 \\
\hline LBNL & ${ }^{107} \mathrm{Ag}$ & 0 & 1039 & 48 & 90 \\
\hline
\end{tabular}

\section{B. Proton Testing}

Proton test data were taken on the Si7414DN at the Massachusetts General Hospital (MGH) Francis H. Burr Proton Beam Therapy Center. The proton beam energy was $200 \mathrm{MeV}$; the scatter beam style was used to randomize the pencil-beam location during proton spills. Individual DUTs mounted to daughter cards were placed at normal incidence and approximately $15 \mathrm{~cm}$ from the beam port in air. Source, drain, and gate voltages were supplied directly to the daughter card by two Keithley 2400 SMUs. In this preliminary test, no stiffening capacitance was added to the drain node. Four DUTs were irradiated to a maximum fluence of $1 \times 10^{10} \mathrm{~cm}^{-2}$ per step in $\mathrm{V}_{\mathrm{DS}}$, with one DUT receiving a total fluence of $1 \times 10^{11} \mathrm{~cm}^{-2}$ at $60 \mathrm{~V}_{\mathrm{DS}}$.

\section{RESULTS}

\section{A. Si7414DN}

\section{1) Heavy-Ion Tests}

Testing was performed with argon and neon ions to evaluate the threshold for catastrophic SEE from galactic cosmic ray ion species with relatively high flux on orbit (i.e., before the "iron knee" of the flux vs. linear energy transfer (LET) curve for galactic cosmic rays (GCRs)). Additional tests with copper and krypton bounded the failure threshold for this higher flux portion of the GCR spectrum. Devices under test were irradiated with $\mathrm{V}_{\mathrm{GS}}$ at $0 \mathrm{~V}$ and $\mathrm{V}_{\mathrm{DS}}$ stepped in $3-\mathrm{V}$ increments per beam run.

Two DUTs were irradiated only at the threshold failure bias condition to confirm that prior beam exposures had not lowered this threshold. DUTs experienced either localized dosing effects (see Fig. 2 for an example) or single-event burnout (SEB). SEB resulted in a resistive pathway between drain and source rather than a complete short, as shown for this same DUT in Fig. 3. In Fig. 4, the BV $\mathrm{B}_{\text {DSs }}$ curves are shown for this DUT prior to irradiation and after the two beam runs. As shown in Fig. 4, cumulative, gradual degradation of the drain leakage current during irradiation resulted in an elevation of drain current at voltages below breakdown, but the voltage at which the characteristic sharp increase in current defining breakdown occurs did not change. In this figure, a solid line marking $64 \mathrm{~V}$ is provided as a guide for comparing the pre- and post- $30 \mathrm{~V}$ irradiation curves. $\mathrm{BV}_{\mathrm{DSS}}$ curve data were taken in one-volt increments at $0 \mathrm{~V}_{\mathrm{GS}}$. Applying the manufacturer $250 \mu \mathrm{A}$ drain current condition, $\mathrm{BV}_{\text {DSS }}$ dropped from $66 \mathrm{~V}$ to under $6 \mathrm{~V}$ after irradiation at $\mathrm{V}_{\mathrm{DS}}=33 \mathrm{~V}$ with $\mathrm{V}_{\mathrm{GS}}=0 \mathrm{~V}$. The corresponding striptape capturing this SEB event is in Fig. 3. To rule out TID effects, $\mathrm{BV}_{\mathrm{DSS}}$ was re-measured 8 months later at $-20 \mathrm{~V}_{\mathrm{GS}}$ and showed no significant change (Fig. 4).

Past research by others has shown that ion strikes through the length of the gate oxide locally shift the flatband voltage, forming a transistor region with lower gate threshold voltage [1]. As shown in this study and in Fig. 5, this shift is worse at non-zero gate bias. Fig. 6 shows the normalized device gate threshold voltage as a function of different ion species. For a given ion species, $\mathrm{V}_{\mathrm{GS}}=0 \mathrm{~V}$ and the DUT $\mathrm{V}_{\mathrm{DS}}$ started at $24 \mathrm{~V}$ and was incremented by $3 \mathrm{~V}$ after each exposure, except for 2 DUTs which were held at $0 V_{D S}$ while exposed to $659 \mathrm{MeV}$ $\mathrm{Cu}$ or $886 \mathrm{MeV} \mathrm{Kr}$ ions (+ and $\times$ symbols). At $0 \mathrm{~V}_{\mathrm{DS}}$, the more pronounced role of interface charge trapping is apparent by the lower rate of change in threshold voltage with increasing dose.

As shown in Fig. 4 after irradiation at $33 V_{D S}$, heavy-ion total ionizing dose also affects the zero-gate drain leakage current $\left(I_{D S S}\right)$. $I_{D S S}$ as a function of total ionizing dose from either $659 \mathrm{MeV} \mathrm{Cu}$ or $886 \mathrm{MeV} \mathrm{Kr}$ is shown in Fig. 7 for the same 2 DUTs irradiated at $0 \mathrm{~V}_{\mathrm{GS}}$ and $0 \mathrm{~V}_{\mathrm{DS}}$ whose shift in gate threshold voltage is given in Fig. 6. Pre-radiation IDSs values were $7 \mathrm{nA}$ and $3 \mathrm{nA}$, respectively. 


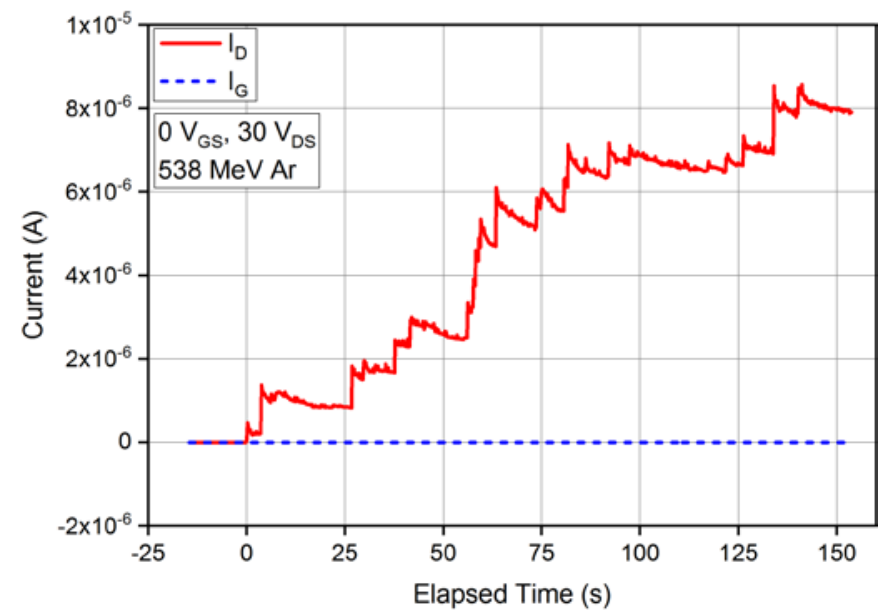

Fig. 2. Si7414DN striptape data showing gate and drain current as a function of time (ion fluence). Ar beam on at $0 \mathrm{~s}$.

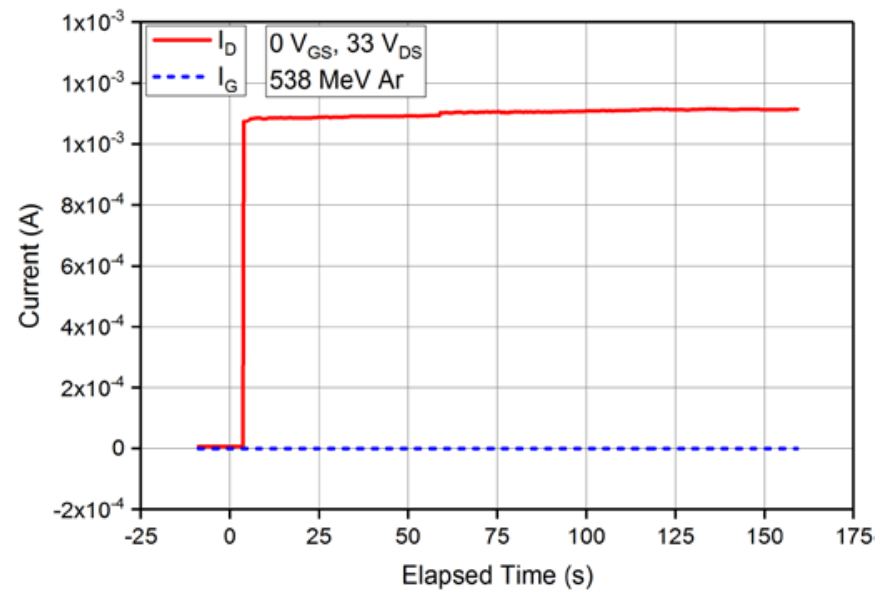

Fig. 3. Si7414DN striptape data showing catastrophic SEB. Drain-source became resistive pathway. Ar beam on at $0 \mathrm{~s}$. This DUT was exposed previously to the beam under a $30 \mathrm{~V}_{\mathrm{DS}}$ bias (see Fig. 2).

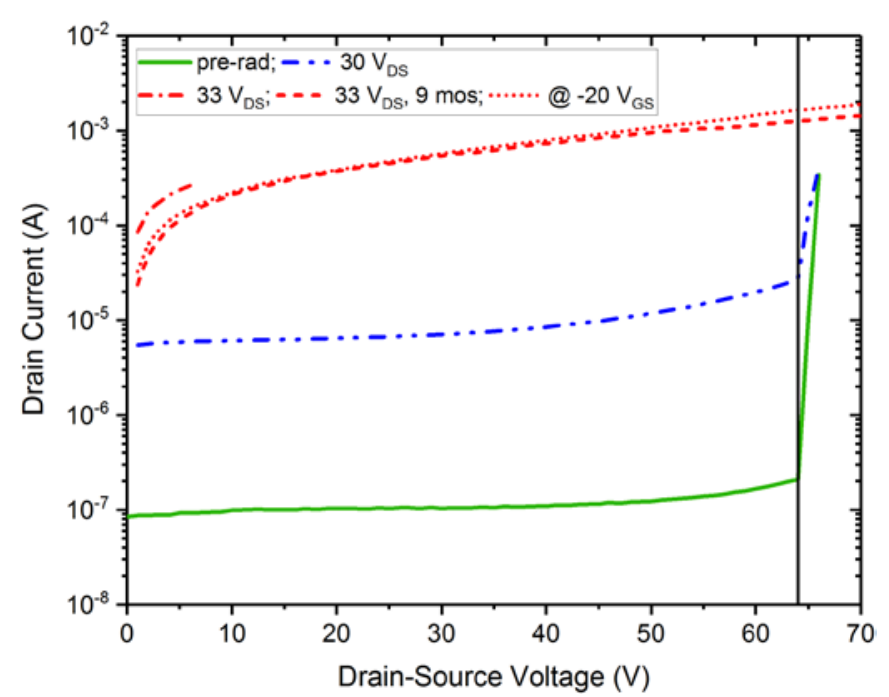

Fig. 4. Si7414DN breakdown voltage curves prior to irradiation and after the two beam exposures at drain voltages indicated in legend. Dashed curve taken 9 months later extends the $33-\mathrm{V}$ post-rad curve to show $\mathrm{ID}_{\mathrm{D}}-\mathrm{V}_{\mathrm{DS}}$ linearity out to higher current/voltage. Dotted line shows BV $V_{\text {Dss }}$ curve is unaffected at hard-off (-20 V) V Vs. Solid vertical black line marks $64 \mathrm{~V}$. $\mathrm{BV}$ DSs is defined by the manufacturer at $250 \mu \mathrm{A}$ drain current.

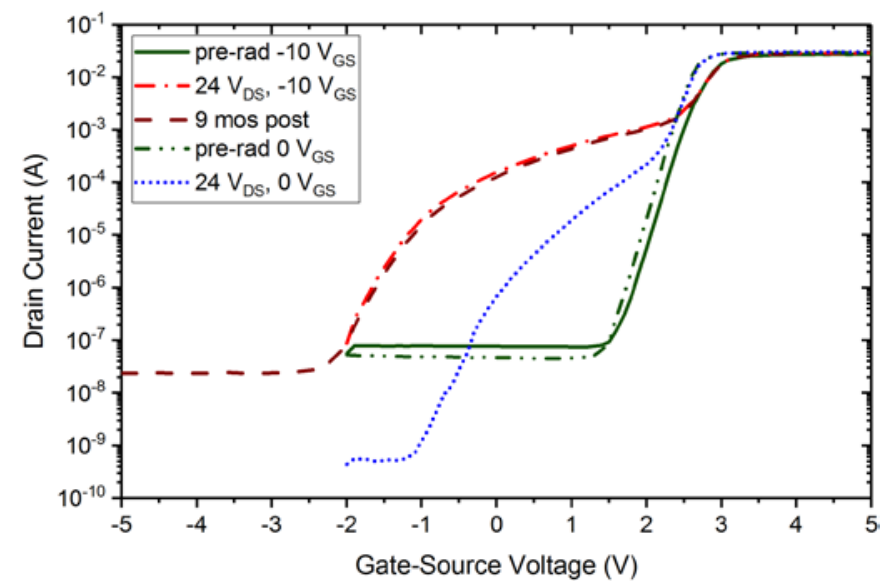

Fig. 5. ID-VGS curves for two Si7414DN DUTs irradiated with $3 \times 10^{6} \mathrm{~cm}^{-2}$ Ar: one at $-10 \mathrm{~V}_{\mathrm{GS}}, 24 \mathrm{~V}_{\mathrm{DS}}$ and the other at $0 \mathrm{~V}_{\mathrm{GS}}$ and $24 \mathrm{~V}_{\mathrm{DS}}$. The minimum off-state current is an artifact of the precision setting of the SMU.

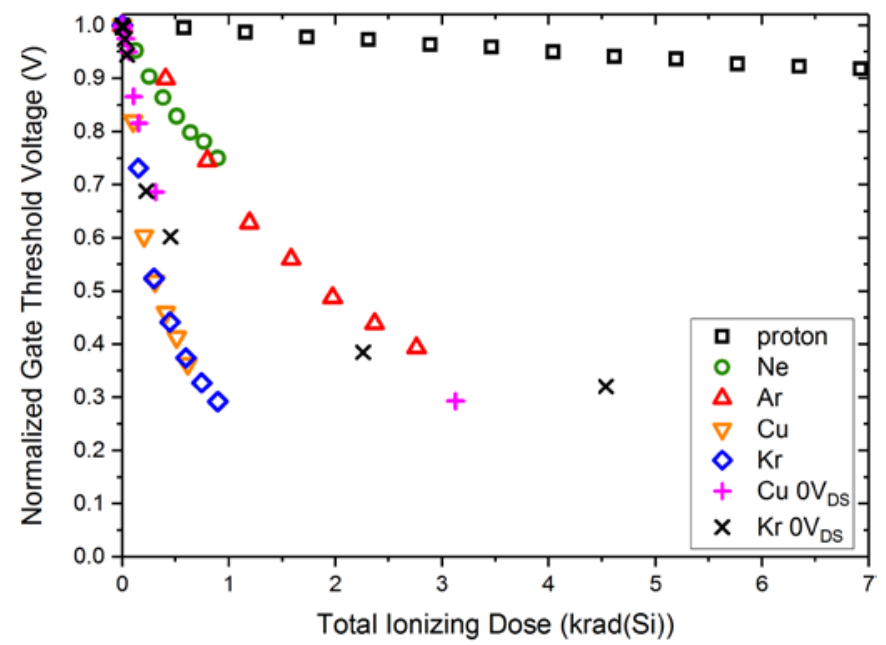

Fig. 6. Normalized gate threshold voltage as a function of total ionizing dose from protons or heavy ions. All parts were irradiated at $0 \mathrm{~V}_{\mathrm{GS}}$. For a given ion species, $\mathrm{V}_{\mathrm{DS}}$ started at $24 \mathrm{~V}$ and was incremented by $3 \mathrm{~V}$ after each exposure, except for 2 DUTs which were held at $0 V_{D S}$ while exposed to $\mathrm{Cu}$ or Kr ions ( + and $\times$ symbols).

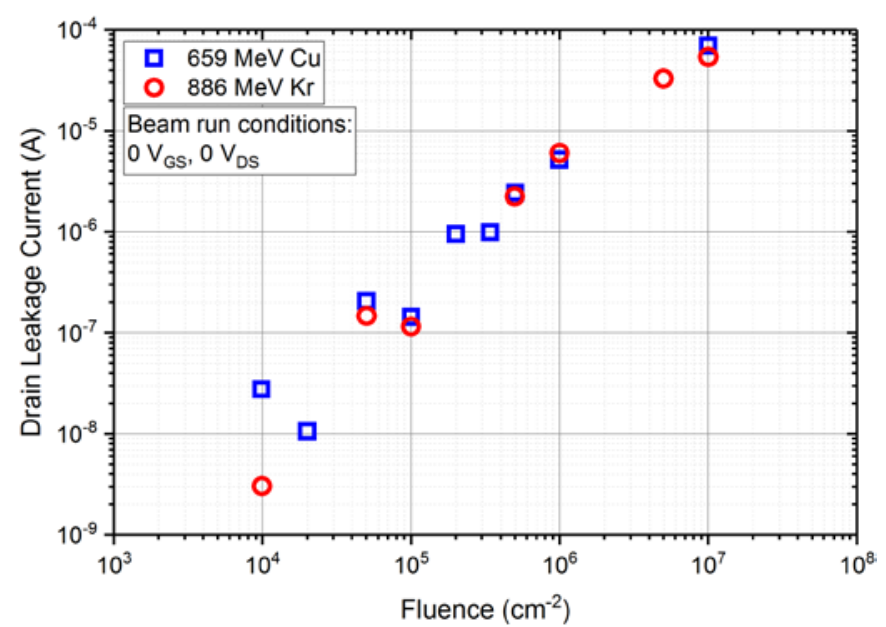

Fig. 7. Zero gate voltage drain leakage current for Si7414DN as a function of fluence for samples irradiated at $0 \mathrm{~V}_{\mathrm{GS}}$ and $0 \mathrm{~V}_{\mathrm{DS}}$. Pre-rad levels were $7 \mathrm{nA}$ $(\mathrm{Cu})$ and $3 \mathrm{nA}(\mathrm{Kr})$. 
A large variability in the threshold voltage for destructive SEE in commercial trench-gate MOSFETs was shown in [9]. Si7414DN also exhibited variability: as shown in SEE threshold voltage versus LET plot in Fig. 8, two DUTs irradiated with argon (LET $=8.2 \mathrm{MeV} \cdot \mathrm{cm}^{2} / \mathrm{mg}$ ) suffered SEB at $45 \mathrm{~V}_{\mathrm{DS}}$, having passed at $42 \mathrm{~V}_{\mathrm{DS}}$. The DUT shown in Fig. 3 failed at only $33 \mathrm{~V}_{\mathrm{DS}}$. An additional DUT failed immediately upon exposure to the $10 \mathrm{MeV} / \mathrm{u}$ argon beam (LET $=9.7$ $\mathrm{MeV} \cdot \mathrm{cm}^{2} / \mathrm{mg}$ ) while at an initial bias condition of $45 \mathrm{~V}_{\mathrm{DS}}$ (indicated in Fig. 8 by the red " $X$ " marker), suggesting that the threshold was not reduced by prior beam exposures. Also shown in Fig. 8, the threshold for SEB under neon irradiation (LET $=2.7 \mathrm{MeV}-\mathrm{cm}^{2} / \mathrm{mg}$ ) was $45 \mathrm{~V}$, based on one DUT, and similar thresholds were found with copper (LET $=21 \mathrm{MeV}$ $\mathrm{cm}^{2} / \mathrm{mg}$ ) and krypton (LET $=31 \mathrm{MeV}-\mathrm{cm}^{2} / \mathrm{mg}$ ).

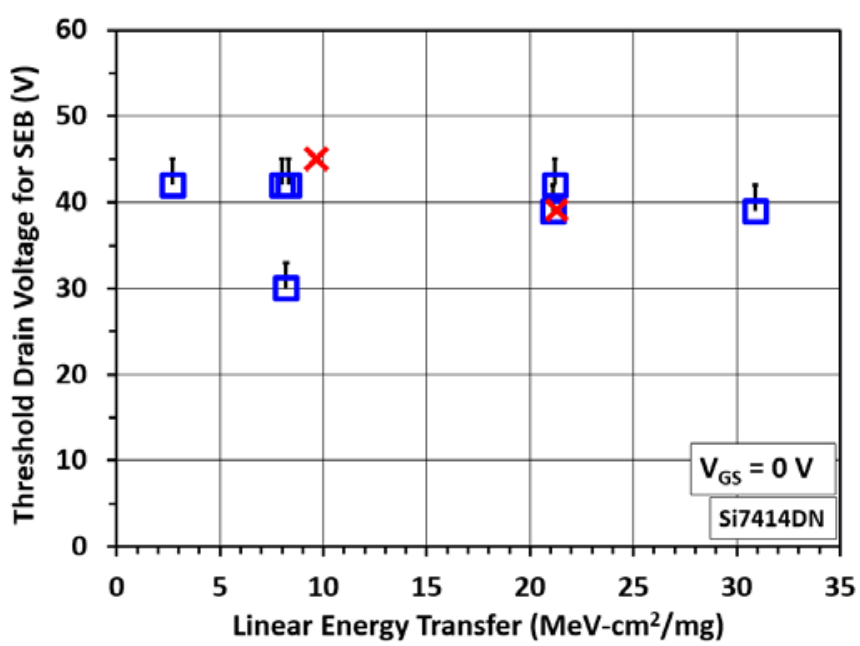

Fig. 8. Si7414DN threshold drain voltage for SEB at $0 \mathrm{~V}_{\mathrm{GS}}$ as a function of LET for individual DUTs. Square markers indicate highest passing voltage; error bars extend to the voltage at which SEB occurred. The $\times$ markers indicate where SEB occurred on the first beam run, thus no prior degradation had accumulated.

\section{2) Proton Tests}

The heavy-ion results showing the threshold for SEB essentially unchanged across the LET and species range evaluated (Fig. 8) raise the possibility of SEB vulnerability from the proton environment. In a preliminary test, four DUTs were irradiated with $200 \mathrm{MeV}$ protons as described in the Methods section above. At $\mathrm{V}_{\mathrm{DS}}$ values below $42 \mathrm{~V}-45$ $\mathrm{V}$, only charge collection was observed during exposure (Fig. 9). At $48 \mathrm{~V}$ for one DUT, and $45 \mathrm{~V}$ for the remaining three, current spikes were measured that increased in frequency (but not measured magnitude) with increased $V_{D S}$ (Figs. 10-11). Note that the peak drain current during these spikes is not known due to the coarseness of the striptape measurements. Additional tests that include drain stiffening capacitance (Fig. 1) in compliance with MIL-STD-750, TM1080 [10], are needed to determine whether these spikes are quenched SEB events, given their similar onset with that of the heavy-ion induced SEB threshold (Fig. 8). Finally, as shown in Fig. 6, post-irradiation characterizations revealed the expected gate threshold voltage decrease from proton ionizing dose; this degradation was less that that from heavier ions.

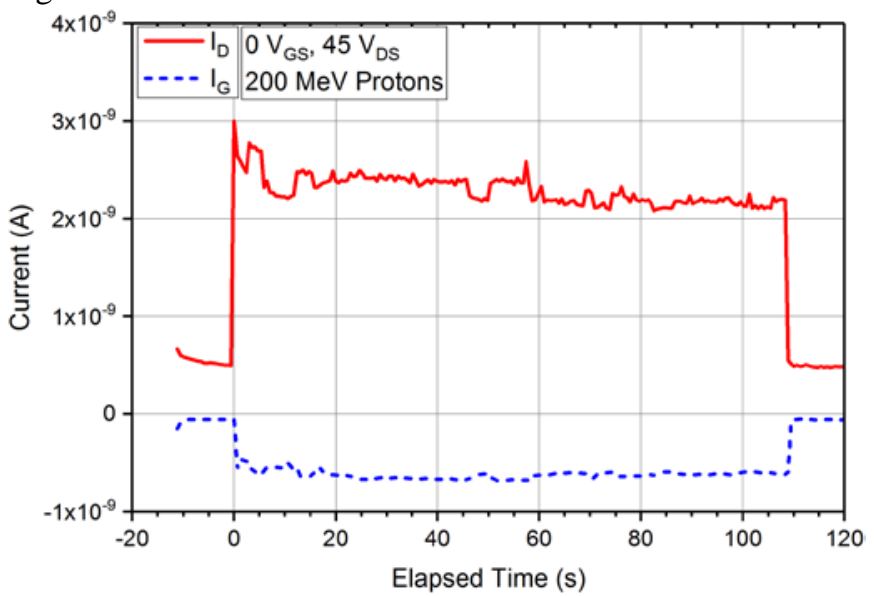

Fig. 9. Charge collection at gate (dashed line) and drain (solid line) nodes of the Si7414DN.

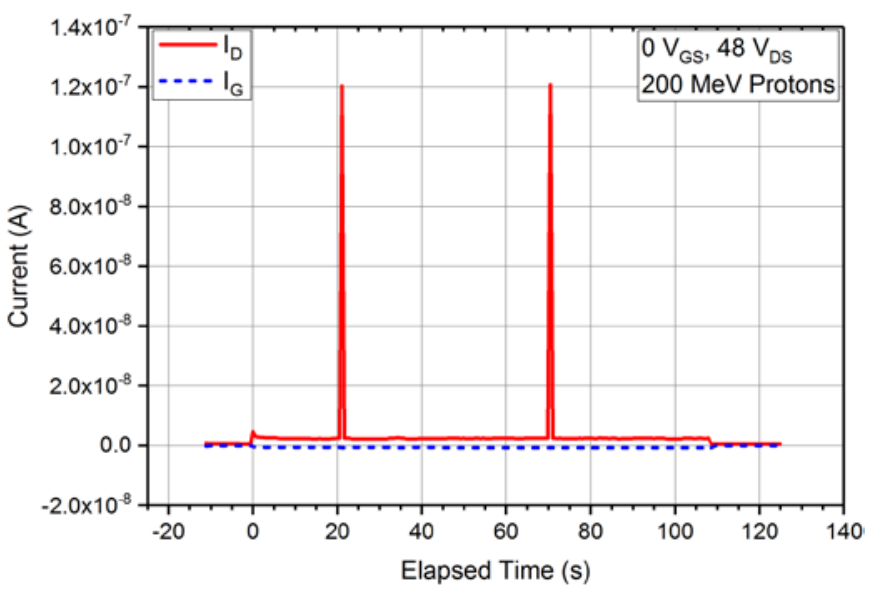

Fig. 10. At $48 \mathrm{~V}_{\mathrm{DS}}$, two drain-current spikes occur during the $200 \mathrm{MeV}$ proton irradiation of the Si7414DN.

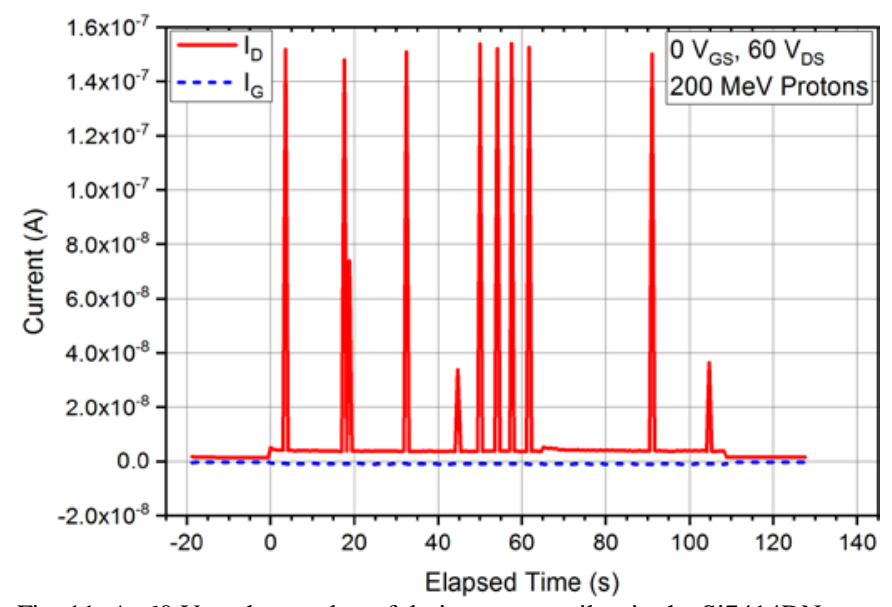

Fig. 11. At $60 \mathrm{~V}_{\mathrm{DS}}$, the number of drain current spikes in the Si7414DN increases but the magnitude is comparable to those at $48 \mathrm{VDS}$ (Fig. 8).

\section{B. SQS460EN}

Testing of the Vishay SQS460EN automotive device was performed with $548 \mathrm{MeV}$ and $400 \mathrm{MeV}$ argon, $659 \mathrm{MeV}$ copper, and $886 \mathrm{MeV}$ krypton. Devices under test were irradiated with $\mathrm{V}_{\mathrm{GS}}$ at $0 \mathrm{~V}$ and $\mathrm{V}_{\mathrm{DS}}$ stepped in $3-\mathrm{V}$ increments 
per beam run. This device demonstrated localized total ionizing dose effects similar to those shown for the Si7414DN. The threshold VDS at $0 \mathrm{~V}_{\mathrm{GS}}$ for SEB is shown in Fig. 12. This device becomes less susceptible to SEB at lower LET and lighter ion species. Although this trend differs from that of the Vishay commercial Si7414DN, it is unlikely to be associated with the grade of the device. Part-to-part variability is roughly commensurate with that seen for the Si7414DN.

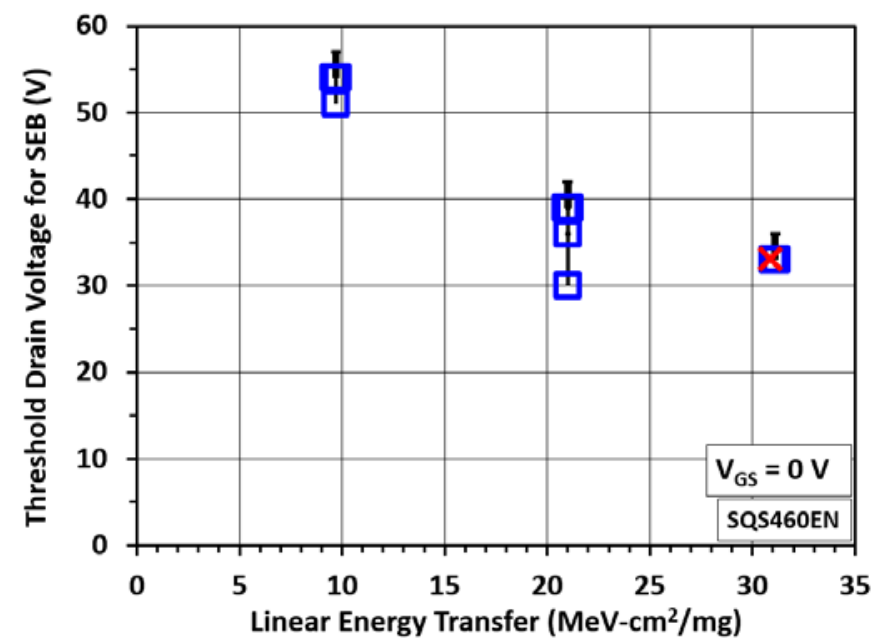

Fig. 12. SQS460EN threshold drain voltage for SEB at 0 VGS as a function of LET for individual DUTs. Square markers indicate highest passing voltage; error bars extend to the voltage at which SEB occurred. The $x$ marker indicates where SEB occurred on the first beam run, thus no prior degradation had accumulated.

\section{SQJ431EP}

The Vishay SQJ431EP trench-gate MOSFET is a -200 V ptype automotive-grade transistor. The localized dosing of the gate oxide in p-type MOSFETs does not impact the overall function of the device because the positive gate threshold shift of the localized area is swamped by overall lower threshold voltage. The failure mode for the p-type unhardened power MOSFETs is typically SEGR. The SQJ431EP exhibited SEGR with $886 \mathrm{MeV}$ krypton irradiation (LET = 31 $\mathrm{MeV} \cdot \mathrm{cm}^{2} / \mathrm{mg}$ ) at $0 \mathrm{~V}_{\mathrm{GS}}$ and $170 \mathrm{~V}_{\mathrm{DS}}$ for 2 DUTs after passing at $160 \mathrm{~V}_{\mathrm{DS}}$; however, the threshold for SEGR was not found due to the third DUT's gate rupturing during its first irradiation at $150 \mathrm{~V}_{\mathrm{DS}}$. Under $548 \mathrm{MeV}$ argon and $659 \mathrm{MeV}$ copper irradiation, all samples passed at the full $-200 \mathrm{~V}$ rated drain voltage. Fig. 13 plots these results. Of the DUTs that failed under krypton irradiation, two failed during the postirradiation gate stress (PIGS) test; the third exhibited two sudden increases in gate leakage current during the beam run (Fig. 14) in addition to failing the PIGS test.

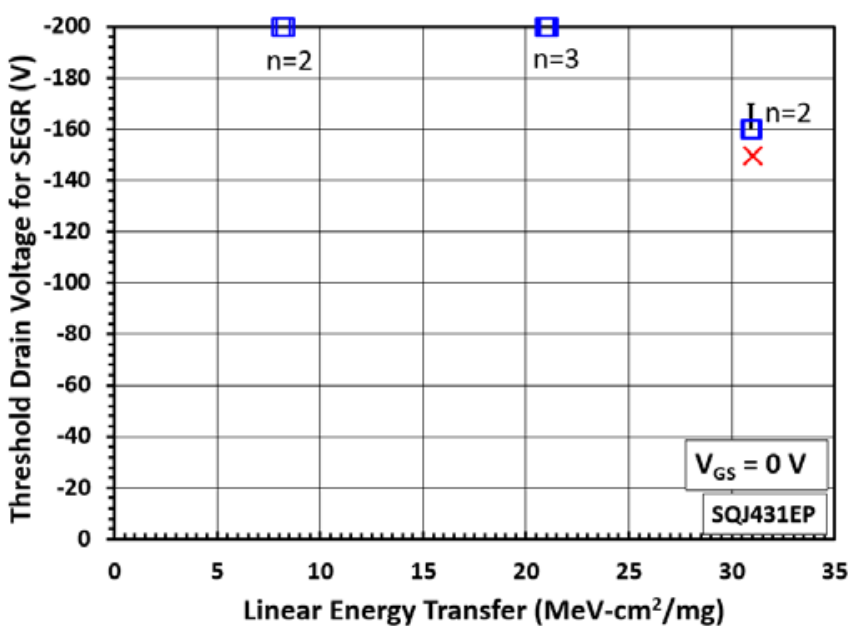

Fig. 13. SQJ431EP drain voltage for SEGR at $0 \mathrm{~V}_{\mathrm{GS}}$ as a function of LET for individual DUTs. Square markers indicate highest passing voltage; error bars extend to the voltage at which SEGR occurred. The $\times$ marker indicates where SEGR occurred on the first beam run; the threshold for SEGR was thus not found.

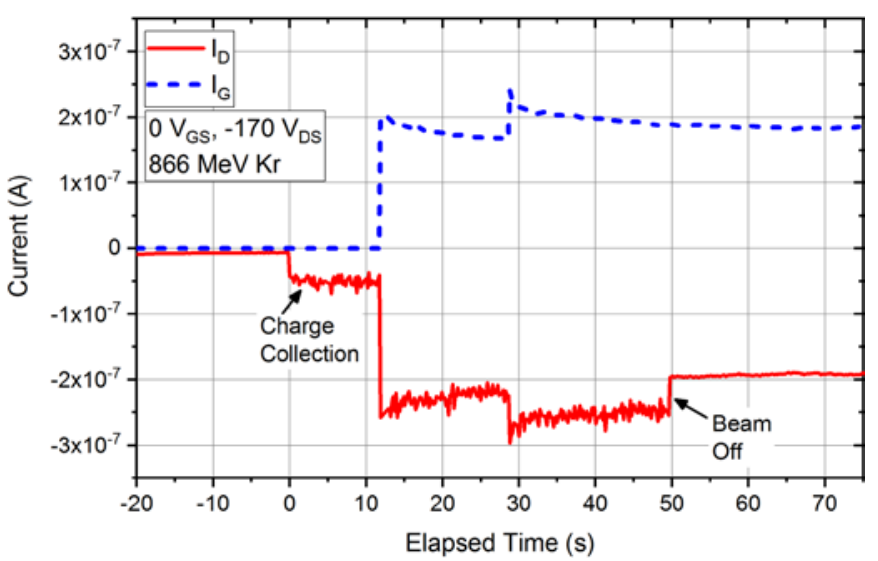

Fig. 14. Striptape of gate (negative) and drain (positive) currents during irradiation of SQJ431EP with krypton. Two small breaks in the gate occurred during this run.

\section{NVTFS5116PL}

The On-Semiconductor -60 V p-type trench-gate power MOSFET passed at $0 \mathrm{~V}_{\mathrm{GS}}$ with $659 \mathrm{MeV}$ copper irradiation but exhibited SEGR during the beam run with $886 \mathrm{MeV}$ krypton irradiation. $\mathrm{V}_{\mathrm{DS}}$ was incremented in 2-volt steps between runs. Fig. 15 shows the individual DUT failure threshold $V_{D S}$ for SEGR as a function of LET, and Fig. 16 provides a striptape showing an example of the gate breakage during the beam run. 


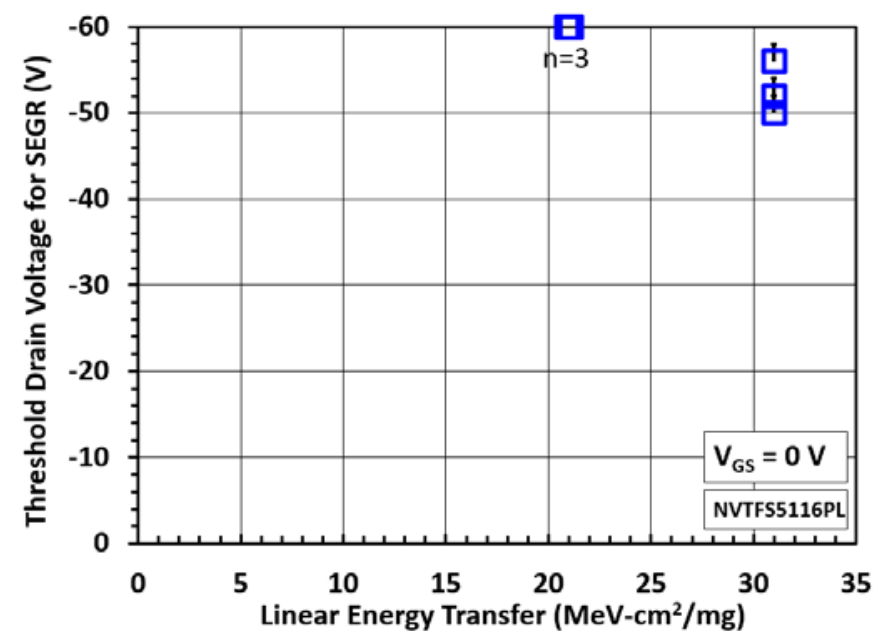

Fig. 15. NVTFS5116PL drain voltage threshold for SEGR at $0 \mathrm{~V}_{G S}$ as a function of LET for individual DUTs. Square markers indicate highest passing voltage; error bars extend to the voltage at which SEGR occurred.

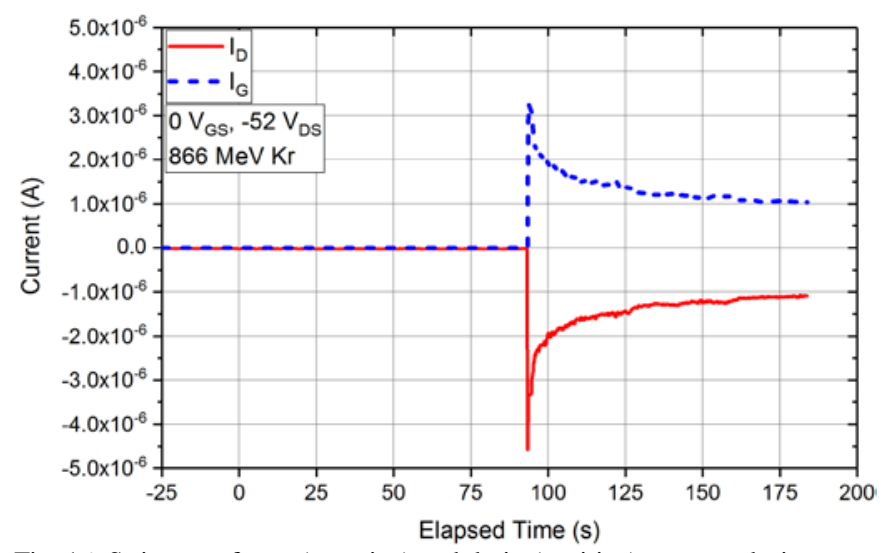

Fig. 16. Striptape of gate (negative) and drain (positive) currents during irradiation of NVTFS5116PL with krypton, showing SEGR.

\section{E. BSS84AKV}

The Nexperia BSS84AKV -50 V p-type trench-gate power MOSFET is a dual power MOSFET whose package dimensions are $1.6 \mathrm{~mm}$ by $2 \mathrm{~mm}$. Due to the small die size, the fluence for each beam run was increased to $1 \times 10^{7} \mathrm{~cm}^{-2}$. The two die within the package are electrically isolated and thus can be individually tested for SEE by selectively biasing one and leaving the other die floating. Samples were irradiated at $0 \mathrm{~V}_{\mathrm{GS}}$ with $\mathrm{V}_{\mathrm{DS}}$ incremented in 2-volt steps. No effects occurred with $659 \mathrm{MeV}$ copper irradiation. As shown in Fig. 17, under $886 \mathrm{MeV}$ krypton, 2 samples passed at -50 $\mathrm{V}_{\mathrm{DS}}$; however, 3 samples exhibited SEGR during the beam run at $-46 V_{D S}$ after passing at $-44 V_{D S}$. There was no correlation between passing or failing samples and whether the die had been previously exposed to the beam while floating.

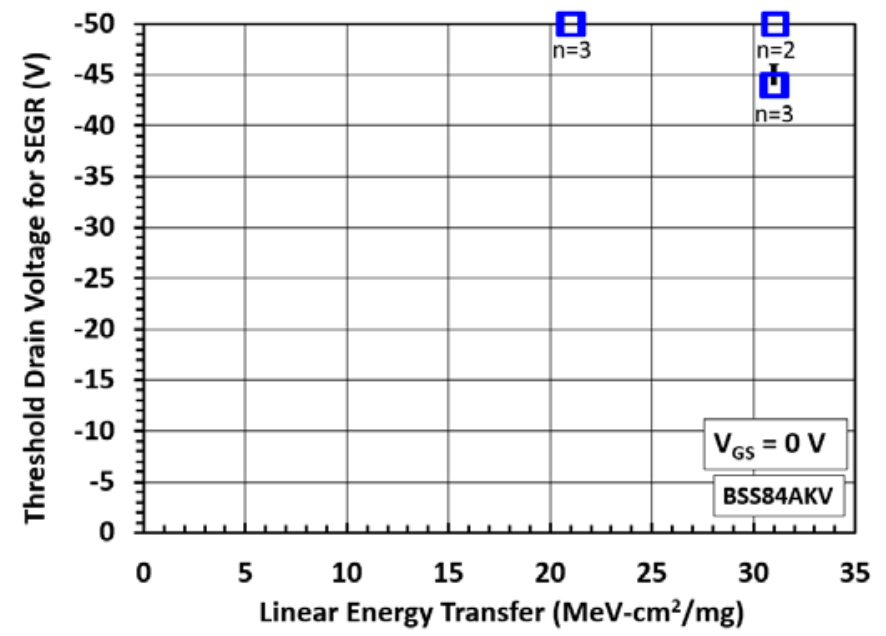

Fig. 17. BSS84AKV drain voltage threshold for SEGR at $0 \mathrm{VGS}_{\mathrm{GS}}$ as a function of LET for individual DUTs. Square markers indicate highest passing voltage; error bars extend to the voltage at which SEGR occurred.

\section{F. IRHLF87Y20}

At this time, only one radiation-hardened trench-gate vertical power MOSFET is available. The Infineon (formerly International Rectifier) generation R8 IRHLF87Y20 is a $20 \mathrm{~V}$ n-type power MOSFET. Irradiation with $1039 \mathrm{MeV}$ silver resulted in no measurable total ionizing dose effects. Fig. 18 shows the SEE threshold $V_{D S}$ as a function of $V_{G S}$, as well as the manufacturer SEE response curve as reported in the data sheet. In this study, $\mathrm{V}_{\mathrm{DS}}$ was incremented in 2-volt steps, and each data point is the result of 3 test samples. Failures were due to either SEB or SEGR. Although there was no part-topart variability in the voltage at which failures occurred, three different failure signatures were seen, demonstrating the greater complexity these trench-gate power MOSFETs have as compared to planar-gate power MOSFETs. The striptape in Fig. 19 shows SEGR occurring during the beam run at 0 $\mathrm{V}_{\mathrm{GS}}$ and $20 \mathrm{~V}_{\mathrm{DS}}$. In Fig. 20, SEB occurs in another sample at these same applied voltage conditions. Finally, in Fig. 21, SEB is seen at $-1 V_{G S}$ and $20 V_{D S}$, but a partial recovery spontaneously occurs. Examination of the drain current in Fig. 20 shows a random telegraph noise-like behavior, suggestive of a charge trapping/detrapping phenomenon.

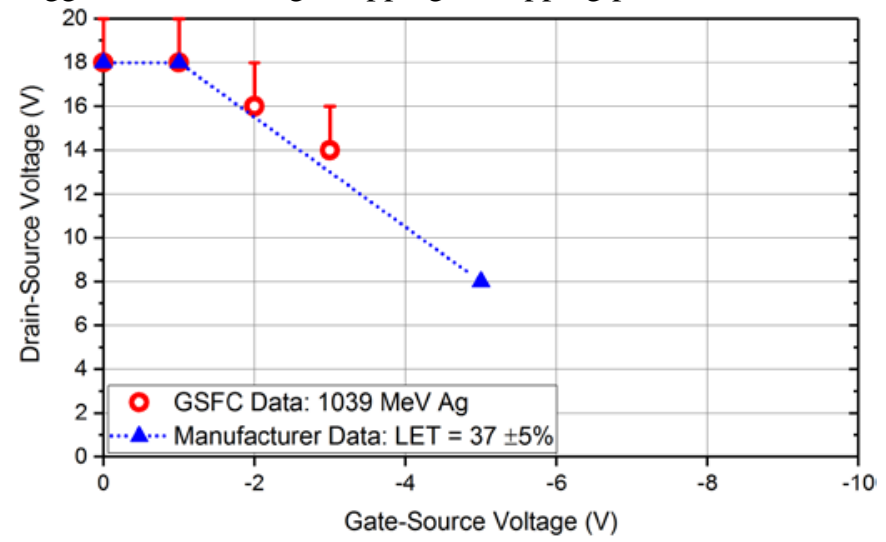

Fig. 18. SEE response curve for IRHLF87Y20 as measured in this study verifies that reported in the manufacturer data sheet. 


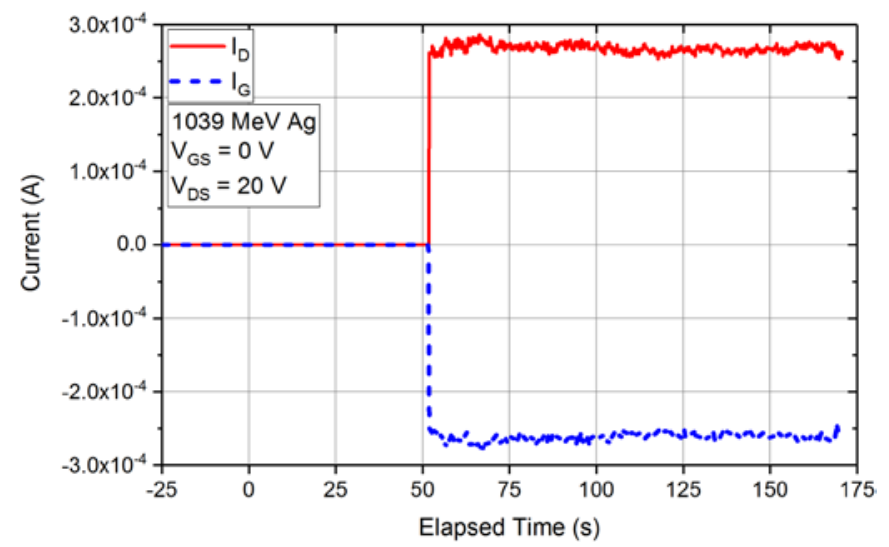

Fig. 19. SEGR occurs during the beam run for IRHLF87Y20 at $0 \mathrm{~V}_{\mathrm{GS}}$ and 20 VDs.

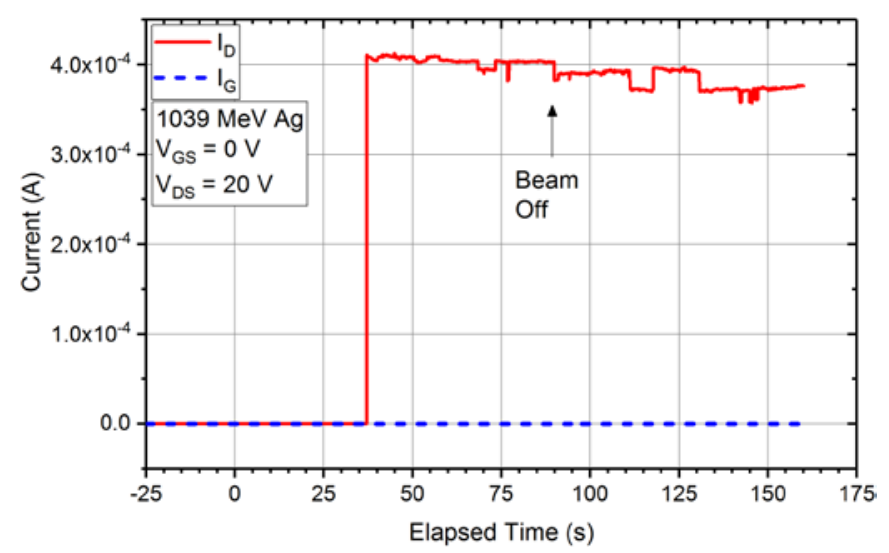

Fig. 20. SEB also occurs in IRHLF87Y20 at 0 VGS and 20 VDS.

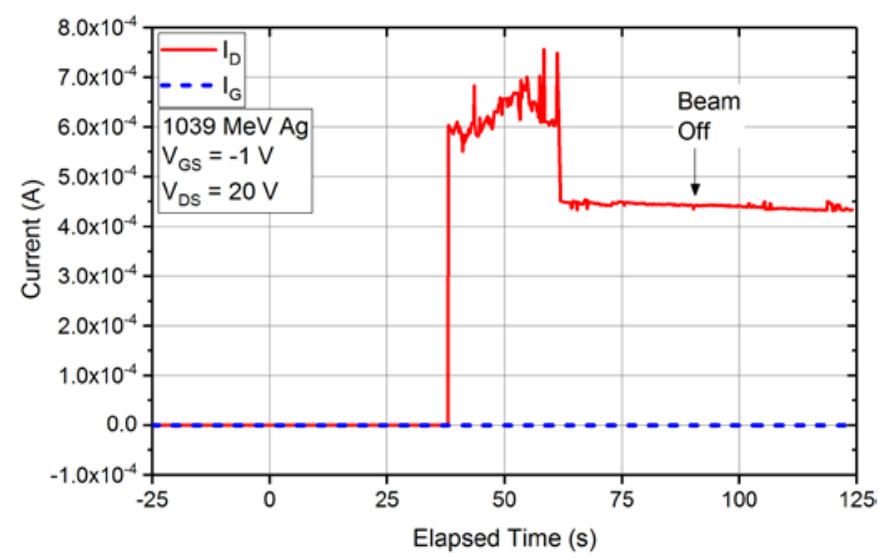

Fig. 21. In this DUT, SEB occurs, followed about 25 seconds later by a partial recover of the drain current.

\section{CONCLUSIONS}

Commercial, automotive, and radiation-hardened trenchgate vertical power MOSFETs were evaluated for SEE sensitivity. The SEE safe operating area for the commercial and automotive-grade devices is difficult to define due to the extent of the part-to-part variability. In some cases, a bimodal distribution may be present (see Fig. 8 , LET $=8.2$ $\mathrm{MeV} \cdot \mathrm{cm}^{2} / \mathrm{mg}$, and Fig. 17, LET $=31 \mathrm{MeV} \cdot \mathrm{cm}^{2} / \mathrm{mg}$ ). A standard radiation hardness assurance procedure is to apply a derating factor (typically 0.75 , per [11]) to the highest passing $V_{D S}$ of the sample that failed at the lowest $V_{D S}$. This approach is likely inadequate given the extent of the part-to-part variability. For example, a 0.75 derating factor applied to the data in Fig. 15 suggests NVTFS5116PL can be operated safely up to a $V_{D S}$ of $37.5 \mathrm{~V}$; however, application of a 99/90 one-sided tolerance limit (KTL) to these data results in $30 \mathrm{~V}$, indicating a larger sample size is needed to determine the distribution of failures.

Hardness assurance is further complicated by the localized dosing effects in the unhardened n-type trench-gate power MOSFETs. As show in Fig. 5, the use of a hard-off VGS bias to counter-act gate threshold voltage shift can increase the amount of shift for a given dose (and potentially create an SEGR susceptibility). As demonstrated in Figs. 6-7, flight spares having all nodes grounded degrade with heavy-ion fluence.

In general, unhardened p-type power MOSFETs tend to be more robust to heavy-ion effects than unhardened n-type power MOSFETs due to the lower mobility of the holes, making SEB unlikely to occur. Instead, SEGR is the typical failure mode. For the devices evaluated in this study, the limited sample sizes suggest that the p-type devices were not susceptible to SEEs from $659 \mathrm{MeV}$ copper ions (LET = 21 $\mathrm{MeV} \cdot \mathrm{cm}^{2} / \mathrm{mg}$ ), whereas the unhardened n-type devices were susceptible to SEEs with $400 \mathrm{MeV}-548 \mathrm{MeV}$ argon (LET = $8.2 \mathrm{MeV} \cdot \mathrm{cm}^{2} / \mathrm{mg}-9.7 \mathrm{MeV} \cdot \mathrm{cm}^{2} / \mathrm{mg}$ ), with one part susceptible to heavy ions as light as neon $($ LET $=2.7$ $\mathrm{MeV} \cdot \mathrm{cm}^{2} / \mathrm{mg}$ ). The susceptibility of the n-type devices to SEB at these low LETs suggests vulnerability to SEB from secondary reaction products when exposed to high-energy protons. In addition, the p-type power MOSFETs experienced SEEs at a higher percentage of their rated $V_{D S}$ as compared to the n-type devices. Finally, the p-type devices do not exhibit the localized dosing effects that impact the unhardened n-type devices when an incident heavy ion ionizes charge along the length of the gate oxide.

At this time, only one radiation-hardened n-type trench-gate power MOSFET is available. This study verified the manufacturer SEE response curve for the IRHLF87Y20. In addition, no localized dosing effects were measured. The device exhibited three different failure signatures during irradiation, demonstrating greater complexity of failure mechanisms than those of planar-gate vertical power MOSFETs.

\section{ACKNOWLEDGMENT}

The authors thank Michael Campola for his work on this project, to Jim Forney and Stephen Cox for their technical assistance, and to Rocky Koga, The Aerospace Corp., for scheduling LBNL beam time for these tests.

\section{REFERENCES}

[1] J. A. Felix, M. R. Shaneyfelt, J. R. Schwank, S. M. Dalton, P. E. Dodd, and J. B. Witcher, "Enhanced degradation in power MOSFET devices due to heavy ion irradiation," IEEE Trans Nucl Sci, vol. 54, pp. 21812189, Dec 2007.

[2] F. K. Galloway, "A brief review of heavy-ion radiation degradation and failure of silicon UMOS power transistors," Electronics, vol. 3, 2014.

[3] S. Kuboyama, A. Maru, N. Ikeda, T. Hirao, and T. Tamura, "Characterization of microdose damage caused by single heavy ion 
observed in trench type power MOSFETs," IEEE Trans Nucl Sci, vol. 57, pp. 3257-3261, 2010.

[4] S. Liu, M. Zafrani, H. Cao, R. Berberian, C. DiCienzo, and M. Boden, "Vulnerable Trench power MOSFETs under heavy ion irradiation," presented at the 2008 IEEE Nuclear and Space Radiation Effects Conference, Tucson, AZ, USA, 14-18 July 2008.

[5] M. R. Shaneyfelt, J. A. Felix, P. E. Dodd, J. R. Schwank, S. M. Dalton, J. Baggio, et al., "Enhanced proton and neutron induced degradation and its impact on hardness assurance testing," IEEE Trans Nucl Sci, vol. 55, pp. 3096-3105, 2008.

[6] G. I. Zebrev, A. S. Vatuev, R. G. Useinov, V. V. Emeliyanov, V. S. Anashin, M. S. Gorbunov, et al., "Microdose induced drain leakage effects in power trench MOSFETs: Experiment and modeling," IEEE Trans Nucl Sci, vol. 61, pp. 1531-1536, 2014.

[7] X. Wan, W. Zhou, D. Liu, H. Bo, and J. Xu, "Charge deposition model for investigating SE-microdose effect in trench power MOSFETs," Journal of Semiconductors, vol. 36, p. 054003, 2015.

[8] "IR's R8 RAD-Hard MOSFETs Increase Efficiency Up to 6\% While Reducing Footprint 50\% compared to Existing Solutions," ed. http://www.infineon.com/cms/en/about-infineon/press/ market-news/2013/irf1449668669059.html, August 15, 2013.

[9] J. S. George, D. A. Clymer, T. L. Turflinger, L. W. Mason, S. Stone, R. Koga, et al., "Response variability in commercial MOSFET SEE qualification," IEEE Trans Nucl Sci, vol. 64, pp. 317-324, 2017.

[10] MIL-STD-750-1A, "Test methods for semiconductor devices," ed: United States Department of Defense, Aug, 2016.

[11] K. Sahu, "EEE-INST-002: Instructions for EEE parts selection, screening, qualification, and derating," NASA/TP-2003-212242, 2003. 